\title{
Competencias digitales docentes para la elaboración de entornos virtuales de aprendizaje
}

\section{Teaching digital skills for the development of virtual learning environments}

\section{Competencias digitales docentes}

José Erazo Delgado. Ing. ${ }^{(1)}$

Carlos Chancay Cedeño. PhD. ${ }^{(2)}$

\section{Vicente Véliz Briones. PhD. ${ }^{(3)}$}

${ }^{1}$ Maestrante en el programa de Maestría de Pedagogía, mención Docencia e Innovación Educativa, Instituto de Posgrado de la Universidad Técnica de Manabí, Portoviejo - Ecuador, jose.erazo@utm.edu.ec

${ }^{2}$ Doctor por la Universidad Autónoma de Madrid. Docente investigador de la Facultad de Filosofía, Letras y Ciencias de la Educación, Departamento de Pedagogía de los Idiomas Nacionales y Extranjeros de la Universidad Técnica de Manabí, Portoviejo - Ecuador, carlos.chancay@utm.edu.ec

${ }^{3}$ Doctor en Ciencias Técnicas. Docente investigador de la Facultad de Ciencias Informáticas, Departamento de Informática y Electrónica. Rector de la Universidad Técnica de Manabí, Portoviejo - Ecuador, vicente.veliz@utm.edu.ec

Contacto: jose.erazo@utm.edu.ec

\section{Resumen:}

Las competencias digitales forman parte del quehacer humano y la educación no se encuentra exenta de ella. Los profesionales de las diferentes áreas deben mantener una constante formación y actualización de conocimientos para mejorar la prestación de sus servicios, más aún el docente que es la persona que se encarga de guiar la enseñanza a sus estudiantes que se encuentran inmersos en el dominio constante de la tecnología. Para esta se realizó una investigación descriptiva con un enfoque 
cualitativo en los docentes de la Facultad de Filosofía, Letras y Ciencias de la Educación de la Universidad Técnica de Manabí a través de las técnicas investigativas como las encuestas, entrevistas y análisis documental. A través de los datos obtenidos se pudo apreciar la importancia de incorporar un programa de formación continua para el fortalecimiento de las competencias digitales del profesorado.

Palabras clave: TIC; EVA; competencias digitales; educación virtual; formación docente

\begin{abstract}
:
Digital skills are part of human endeavor and education is not exempt from it. The professionals of the different areas must maintain a constant and updating of knowledge to improve the provision of their services, even more so the teacher who is the person who is in charge of guiding the teaching of their students who are immersed in the constant mastery of the technology. For this, a descriptive research was carried out with a qualitative approach in the teachers of the Faculty of Philosophy, Letters and Education Sciences of the Technical University of Manabí through investigative techniques such as surveys, interviews and documentary analysis. Through the digital data obtained, it was possible to appreciate the importance of incorporating a continuous training program to strengthen the skills of teachers.
\end{abstract}

Keywords: TIC; EVA; digital skills; virtual education; teacher training

\title{
Introducción
}

Acorde transcurren los años van surgiendo cambios en los sistemas educativos, tales como la incorporación de nuevas modalidades de estudios relacionadas a la virtualidad. Por ello, el docente como eje dinamizador de la educación debe actualizarse constantemente en el uso de las herramientas digitales. Se deben mejorar las destrezas en competencias digitales que permitan crear estrategias educativas para desarrollar en los estudiantes su pensamiento crítico, reflexivo y creativo, y con ello el mejoramiento del rendimiento académico.

Es importante brindar una educación acorde a las necesidades de los estudiantes actuales, mediante el uso de trabajo autónomo y cooperativo, en ambientes que requieran herramientas virtuales o no, 
propias a la edad de los educandos. Para lograr esto el docente debe estar actualizado con información de la asignatura que imparte, también debe capacitarse en el uso y manejo de las Tecnologías de la Información y Comunicación que la educación actual exige.

A nivel global el uso de entornos virtuales de aprendizaje no es algo nuevo, forma parte de los proceso de enseñanza-aprendizaje. El uso de los entornos virtuales de aprendizaje no solo permite mejorar el proceso pedagógico, sino que permite una comunicación con el estudiante desde cualquier lugar y hora. Esta situación obliga al personal docente a formarse en entornos digitales de aprendizajes siendo el Moodle es más utilizado para proporcionar a sus estudiantes una educación de calidad, con la utilización de recursos que están acostumbrados a utilizar en su vida cotidiana para fomentar el ocio, pero esta vez para generar un contexto pedagógico ideal.

El objetivo de esta investigación fue recabar información necesaria sobre el conocimiento que posee el profesorado acerca de las competencias digitales, y posteriormente poder llevar a la práctica capacitaciones según su nivel de conocimiento que les permitan la utilización de recursos que van desde la creación de tareas, subida de documentos, creación de cuestionarios, generación de reactivos, videoconferencias, entras otras actividades importantes.

Se trabajó con información obtenida de los docentes de la Facultad de Filosofía, Letras y Ciencias de la Educación para corroborar datos y conocer estado de las competencias digitales del profesorado, misma que servirá como guía para crear las estrategias adecuadas para que sean comprendidas por el personal docente.

\section{Materiales y métodos.}

La presente investigación tiene carácter descriptivo con el uso del enfoque cualitativo. Se utilizó un diseño etnográfico educativo aplicado a los docentes de la Facultad de Filosofía, Letras y Ciencias de la Educación. Para la selección de los participantes en la investigación se realizó un muestreo no probabilístico mediante el efecto bola de nieve. Se aplicaron tres instrumentos de recolección de datos: (1) la encuesta (cuestionario de preguntas), (2) la entrevista (guía de preguntas) y (3) el análisis de contenido (ficha de contenidos). La encuesta proviene de la tesis doctoral La formación en 
competencias digitales de los estudiantes del magisterio de la Universidad Autónoma de Madrid. La entrevista fue validada por expertos en el área de la tecnología educativa.

\section{Resultados}

Análisis de la encuesta aplicada a los docentes de la Universidad Técnica de Manabí.

\section{Tabla 1: Recursos tecnológicos}

\begin{tabular}{lc}
\hline Recursos tecnológicos & Total \\
\hline Ordenador de sobremesa & 12 \\
\hline Ordenador portátil & 37 \\
\hline Conexión a Internet & 39 \\
\hline Smartphone & 28 \\
\hline Tablet & 8 \\
\hline Pizarra Digital Interactiva & 0 \\
\hline Videoconsola & 1 \\
\hline Ninguno de ellos & 1 \\
\hline Otras & 0 \\
\hline
\end{tabular}

El principal recurso tecnológico con el que cuenta los docentes es el internet, este recurso lo complementa con el uso de ordenadores y Smartphone. Además, se puede observar que cada uno cuenta con dos o más de los recursos mencionados en esta interrogante, que posean varios recursos tecnológicos confirma que tienen las herramientas necesarias para enfrentar los nuevos retos de formar estudiantes en la sociedad red

\section{Tabla 2: Uso de las TIC}




\begin{tabular}{lcc}
\hline Uso de las TIC & Total & Porcentaje \\
\hline Utilizo las TIC en casa como entretenimiento & 24 & $61.53 \%$ \\
\hline Utilizo las TIC en casa como medio de formación & 14 & $35.89 \%$ \\
\hline No uso las TIC en casa & 1 & $2.56 \%$ \\
\hline Otros & & $0 \%$ \\
\hline
\end{tabular}

La mayoría de los participantes utilizan las TIC en casa como medio de entretenimiento, y no explotan el potencial del uso de las TIC como medio de formación. Para ello, Errrobidart (2019) menciona la importancia de la incorporación, no solo como medio de entretenimiento, sino como una fuente de formación del profesorado para poder potenciar la educación en la ciudadanía digital

\section{Tabla 3: Formación para uso de TIC}

\begin{tabular}{lc}
\hline Formación para uso de TIC Total & TC
\end{tabular}

He recibido formación expresa en el uso de las TIC 36

He realizado formación autónoma en el ámbito de las TIC 30

No he recibido formación, pero me gustaría

0

No tengo interés en formarme

0

Otros

0

Según la información obtenida los participantes han tenido formación en el uso de las TIC, se logra apreciar que los docentes poseen el interés por formarse en competencias digitales, esta teoría toma más fuerza al observar que ningún docente escogió las opciones de no he recibido formación pero me gustaría o no tengo interés en formarme. Para ello, Chou et al. (2016) menciona que los procesos 
educativos son cambiantes, eso ocasiona nuevos retos para los docentes en los procesos académicos, los cuales están vinculados con el uso de herramientas digitales.

\section{Tabla 4: Herramientas web y de creación de recursos multimedia}

\begin{tabular}{|c|c|c|}
\hline \multirow{3}{*}{ Preguntas } & \multicolumn{2}{|l|}{ Rango } \\
\hline & Poco capaz & Muy capaz \\
\hline & 1 & 5 \\
\hline 12)Modificar imágenes mediante algún programa de diseño gráfico & 2.94 & \\
\hline $\begin{array}{l}\text { 13)Resolver problemas como configurar un correo electrónico, configurar y } \\
\text { actualizar un antivirus. }\end{array}$ & 3.46 & \\
\hline $\begin{array}{l}\text { 14)Crear un clip de audio con algún programa informático y convertirlo en } \\
\text { PodCast }\end{array}$ & 2.92 & \\
\hline $\begin{array}{l}\text { 15)Realizar un documento con un procesador de texto, usando técnicas com } \\
\text { oencabezamiento, gráficos, textos de diferentes formatos. }\end{array}$ & 3.94 & \\
\hline 16) Uso el sistema de sindicación (RSS) & 2.56 & \\
\hline 17) Diseñar, crear y modificar Blogs y Páginas Web & 2.87 & \\
\hline 18) Realizar búsquedas avanzadas en los diferentes buscadores & 3.76 & \\
\hline $\begin{array}{l}\text { 19)Navegar por Internet con diferentes exploradores, borrar historial, cambi } \\
\text { ar la configuración del explorador... }\end{array}$ & 3.74 & \\
\hline 20) Coordinar una actividad en grupo realizada a través de Internet. & 3.92 & \\
\hline 21) Realizar videoconferencias por IP (Skype, Zoom...) & 4.15 & \\
\hline 22) Realizar actividades en la plataforma Jclic o HotPotatoes & 2.38 & \\
\hline
\end{tabular}


23)Subida y envío de ficheros a traves de programas FTP

24)Realizar mapas conceptuales multimedia

25) Crear páginas web con texto, imágenes, links...

26) Realizar presentaciones multimedia

27) Realizar actividades educativas con realidad aumentada

TOTAL
2.89

3.43

2.94

3.46

2.69

A partir del análisis de la tabla $n^{\circ} 4$ el claustro indico tienen un nivel básico del uso de herramientas digitales y ofimática. Para la interpretación de los datos de la interrogante 12 hasta la 27, se utilizó la escala de Likert como técnica de medición, con una valoración desde 1 (poco capaz) hasta 5 (muy capaz). Para obtener los resultados en cada pregunta, se sumaron las cantidades logrando identificar un valor medio.

Estos resultados confirman que la mayoría de los docentes poseen un nivel básico en el uso de herramientas digitales. No se debe olvidar que la sociedad avanza de forma acelerada junto con la tecnología, para ello, es importante que los docentes tengan un programa de capacitación continua para brindar una buena experiencia educativa a la ciudadanía digital. Los resultados mostraron que los docentes poseen un mayor dominio en el uso de herramientas web en comparación a las herramientas de creación de recursos multimedia. Para García (2011) y Balladares-Burgos (2018) el docente no es solo un trasmisor de conocimientos específicos, sino es un creador de ambientes de aprendizajes con la capacidad de rentabilizar los espacios ya sean de modalidad presencial o virtual donde se produce la praxis docente.

\section{Análisis de entrevista aplicada a los docentes de la Universidad Técnica de Manabí.}

En la entrevista aplicada a los docentes de la Facultad de Filosofía, Letras y Ciencias de la investigación se pudo apreciar información importante del manejo y uso de las herramientas digitales 
en la práctica docente. En este sentido, han adquirido un dominio de herramientas TIC básicas para el desarrollo del proceso de enseñanza aprendizaje que gira mayormente en el uso del infocus (ver cita e1:p4 y e17:p4 debajo). Los docentes piensan que el uso del power point es un medio para innovar y no es así, puesto que el infocus solo tiene la función proyectar contenidos (ver cita e7:p4 y e15:p4 debajo), y son esos contenidos los que se necesitan elaborar a través de las TIC.

- En trabajos presenciales mediante proyecciones, y ahora en lo virtual utilizando varias herramientas y recuerdos que me han permitido tener un mejor acercamiento con el estudiante (e1:p4)

- Mediante los vídeos con las clases, utilizando el powert point, lecturas relacionadas al tema. (e7:p4)

- Utilizo frecuente presentaciones powerpoint (e15:p4)

- Usando presentaciones con Infocus (e17:p4)

Uno de los aspectos a mejorar es el uso de herramientas digitales por parte del claustro docente, cabe mencionar, que en su mayoría solo asisten a capacitaciones realizadas por la misma universidad (ver cita e10:p5 y e11:p5 debajo), y que muchas veces estas resultan ser repetitivas, en este caso, los participantes creen necesario e importante participar en capacitaciones interinstitucionales (e21:p5 y e5:p5 debajo) para generar un intercambio de conocimientos y compartir experiencias de diferentes contexto con relación a las herramientas TIC existentes.

- Por webinar y capacitaciones de la Facultad (e10:p5)

- Por capacitaciones de la universidad. (e11:p5)

- Con capacitaciones externas e internas de la universidad (e21:p5)

- Lectura, autoformación, cursos de posgrado que he cursado (e5:p5)

Los docentes utilizan como principales medios para generar trabajo colaborativo las herramientas prezi y zoom (ver cita e11:p3 y e14:p3 debajo), y al ser de uso constante resulta desmotivador para 
los estudiantes, esto causa poco interés para realizar actividades. Sumado a esto, los docentes usan metodologías de clase invertida y aprendizaje basado en proyectos (ABP) como fuente de innovación (ver cita e4:p1 y e5:p1 debajo), pero al no tener el dominio adecuado de la tecnología en tiempos de virtualidad, no se logran los objetivos deseados y muchas ocasiones no se logran los resultados propuestos para la actividad.

- Reuniones vía Zoom y Mitents porque son herramientas que permiten interactuar en tiempo real (e11:p3)

- (utiliza como herramientas de trabajo colaborativo) Foros y prezzi. (e14:p3)

- Aula invertida, proyectos, aprendizaje cooperativo (e4:p1)

- Proyectos. aprendizaje cooperativo, aula invertida (e5:p1)

Sumado a esto, reconocen poseer los medios necesarios para crear y diseñar el proceso de enseñanza aprendizaje, pero al no contar con el conocimiento necesario se vuelven un problema latente para los docentes. La plataforma Moodle les resulta interesante pero debido a la falta de conocimientos en competencias digitales se les dificulta crear contenido (ver cita e16:p2 debajo), realizar configuraciones del calificador (ver cita e1:p2 debajo) y tareas necesarias (ver cita e9:p2 debajo) para otorgar roles a los estudiantes (ver cita e9:p2 debajo) y asentar calificaciones que evidencien el cumplimiento de estas.

- Configurar las calificaciones (e1:p2)

- Que la información no llegue de la misma manera a los estudiantes. (e9:p2)

- Configurar las tareas con imágenes en la descripción (e19:p2) 
- Problemas en la configuración del aula (e16:p2)

Esta información demuestra la preocupación de los docentes por adquirir nuevos conocimientos relacionados al uso de herramientas TIC que les permita realizar su praxis docente en la sociedad red.

\section{Análisis de documental a los docentes de la Universidad Técnica de Manabí.}

Mediante el análisis exhaustivo de syllabus y guías de estudio que utilizan los docentes de las modalidades presenciales y online de la Facultad de Filosofía, Letras y Ciencias de la Educación, se pudo apreciar como combinan las actividades con recursos tecnológicos, lo que demostró un poco uso de herramientas TIC en su praxis docente, principalmente en los docentes de la modalidad presencial. Asimismo, se logró observar que la mayoría de syllabus no contaban con la descripción de las actividades aplicadas, estos documentos en su mayoría mostraban solo el uso de foro, test y tarea como recursos dentro del Moodle.

El Moodle es una plataforma que cuenta con múltiples herramientas para mejorar la praxis docente, herramientas como LTI, H5P, actividad taller, entre otras, han sido omitidas dentro de documentos que poseen la planificación docente y son muy importante para mejorar la interactividad del docente hacia sus estudiantes, siendo necesario considerar porque son herramientas con las que cuentan los docentes.

\section{Discusión}

Según Almerich Cerveró et al. (2011) el claustro docente mantiene niveles medios y bajos de competencias digitales, y los recursos utilizados en entornos personales siguen siendo mayores a la tecnología utilizada en entorno educativos. Para ello, Arancibia et al. (2020) en sus investigaciones demuestra que los docentes con tendencias constructivistas tienen un mayor dominio de entornos virtuales de aprendizaje, mientras que los docentes con tendencias conductistas tienen un dominio bajo de herramientas digitales. Por consiguiente, se aprecia una mayor resistencia del manejo de recursos digitales por parte de los docentes con tendencias conductistas, esto se debe a que los medios digitales tienen una mayor tendencia al enfoque constructivista al momento de realizar la praxis 
docente. Asimismo, (Hernández et al., 2014) menciona que el uso de las TIC en la educación se favorece cuando se tiene al alto dominio técnico y no solo pedagógico al momento de efectuar las practicas académicas. Por tal razón, es importante incorporar programas de capacitación continua para favorecer la praxis docente mediada por las TIC.

Balladares-Burgos (2018) expresa que la Educación Superior ha incorporado el uso de las TIC en sus procesos de enseñanza-aprendizaje tanto dentro como fuera del aula. Estos cambios se realizan con el afán de mejorar la calidad educativa, y el dominio de herramientas digitales debe figurar como parte de las competencias del docente, estas deben fundamentarse tanto en la docencia, gestión académica y vinculación con la sociedad

De manera especial Chou et al. (2016) piensan que las instituciones de educación superior deben implementar en los procesos formativos elementos TIC; recursos, contenidos digitales, aplicaciones, y herramientas de la web 2.0. Como resultado, tales herramientas se vuelven agentes de cambio, capaces de mejorar los procesos de formación educativa en comparación a los procesos tradicionales, desde la gestión, la metodología, la evaluación, entre otros. Al respecto conviene decir que Marín et al. (2012), el principio del siglo XXI ha traído, entre otros aspectos una forma diferente de ver, sentir y comprender el rol del docente universitario; las metodologías utilizadas, así como la forma de interpretar los procesos educativos, que se deben diseñar desde una perspectiva distinta y a su vez nueva. El docente debe asumir un nuevo rol de guía en el proceso de selección de la información que la red facilita a los estudiantes durante el proceso de formación.

Los autores Chou (2016) y Marín (2012) indican que el cambio constante en la sociedad afecta a la educación y a la forma de aprender de los estudiantes, esto origina que los docentes deban enfrentar nuevos retos para mantenerse al día con los procesos académicos que se requieren en la actualidad, vinculando las herramientas digitales como medio de formación. La renovación pedagógica digital del docente toma un papel fundamental para mejorar los procesos educativos, quienes deben aprender, mejorando lo aprendido y en algunos casos desprender Agreda et al. (2016).

Como es conocido, los avances fundamentales y la proyección actual de los modelos de formación virtual se deben en gran medida a la incorporación de las Tecnologías de la Información, las comunidades y a elementos pedagógicos provenientes de teorías socioculturales del aprendizaje. Las 
TIC han favorecido el desarrollo de estos enfoques porque ofrecen un soporte práctico para la interacción entre estudiante-profesor y entre los propios estudiantes así como la construcción colectiva de conocimiento. Los entornos virtuales de aprendizaje han ayudado a los modelos pedagógicos centrados en la transmisión de saberes que se basan en el aprendizaje y la construcción de conocimiento. Esta concepción supone que los estudiantes se conviertan en agentes activos del proceso de aprendizaje y los profesores se transformen en orientadores o tutores en la construcción apropiación de conocimientos. Estrada et al. (2015)

Los entornos virtuales aprendizaje son espacios en línea propios del siglo XX, transformándose en unos de los desafíos de la educación actual, sobre todo en los docentes que vienen ejerciendo la docencia desde años remotos. Asumir el rol de docente es prepararnos en el manejo de estrategias y herramientas tecnológicas que en la actualidad son necesarias para crear una sociedad digital. Martín et al., (2019) indica, que muchas universidades están apoyando el uso de los entornos virtuales de aprendizaje como herramienta fundamental en la educación presencial, debido a que permite flexibilizar e individualizar en la educación. Por otro lado Pablo González (2017), el uso de la tecnología está contribuyendo a elaborar nuevos escenarios de aprendizaje en la educación, mediante el entorno virtual de aprendizaje (EVA) se permite innovar en la forma que se aprende.

\section{Conclusión}

A continuación, se concluye que las competencias digitales en el profesorado son necesarias para afrontar el ejercicio docente en tiempos de pandemia. Asimismo, estas competencias coadyuvan a que el profesorado pueda desarrollar sus clases con el apoyo de las tecnologías aplicadas al campo educativo. Se debe crear un proceso de formación continua para el profesorado, debido que los estudiantes del mañana aprenden de forma diferente por su relación con los recursos tecnológicos e incluir las TIC en el proceso de enseñanza- aprendizaje ayudara a crear un aprendizaje más duradero con el tiempo a través de actividades diseñadas para cada contexto educativo.

\section{Referencias bibliográficas}

Agreda, M., Hinojo, M. J., \& Sola, M. J. (2016). Páginas 39 a 56 Píxel-Bit. Píxel-Bit Revista de Medios y Educación, 03(49), 39-56. https://doi.org/10.12795/pixelbit.2016.i49.03 
Almerich Cerveró, G., Suárez Rodríguez, J. M., Jornet Meliá, J. M., \& Orellana Alonso, M. N. (2011). Competencies and the Use of Information and Communication Technologies (ICT) by the Teaching Staff: Dimensional Structure. Competencies and the Use of Information and Communication Technologies (ICT) by the Teaching Staff: Dimensional Structure, 13(1), 2842.

Arancibia, M. L., Cabero, J., \& Marín, V. (2020). Creencias sobre la enseñanza y uso de las tecnologías de la información y la comunicación (TIC) en docentes de educación superior. Formación Universitaria, 13(3), 89-100. https://doi.org/10.4067/s0718-50062020000300089

Balladares-Burgos, J. A. (2018). Diseño pedagógico de la educación digital para la formación del profesorado Instructional design of digital education for teacher training. Revista Latinoamericana de Tecnología Educativa, 17(1), 41-60. https://doi.org/10.17398/1695288X.17.1.41

Chou, R., Valdés, A., \& Sánchez, S. (2016). Programa de formación de competencias digitales en docentes universitarios. Revista Científica Universidad y Sociedad, 8, 81-86.

Pablo González, G. (2017). Factores que favorecen la presencia docente en entornos virtuales de aprendizaje. Tendencias Pedagógicas, 29(2017), 43-58.

https://doi.org/10.15366/tp2017.29.001

Errrobidart, A. (2019). La comunicación pedagógica y las TIC en la escuela secundaria: misceláneas de una relación conflictiva. Educación, Lenguaje y Sociedad, XVII(17), 1-18.

Estrada, V., Febles, J., Passailaigue, R., Ortega, C., \& Mendoza, M. (2015). La educación virtual.

García, C. M. (2011). Profesorado. Revista de Currículum y Formación de Profesorado.

Hernández, L., Acevedo, J., Martínez, C., \& Cruz, B. (2014). El uso de las TIC en el aula: un análisis en términos de efectividad y eficacia. Congreso Iberoamericano de Ciencia, Tecnología, Innovación y Educación, 1-21.

Marín, V., Vázquez, A., Llorente, C., \& Cabero, J. (2012). La Alfabetización Digital Del Docente 
Universitario en el espacio Europeo de educación superior. Edutec. Revista Electrónica de Tecnologia Educativa, 39, 1-10.

http://edutec.rediris.es/Revelec2/Revelec39/alfabetizacion_digital_docente_universitario_E EES.html $\% 5 \mathrm{Cn} 10$

Martín, A. C. U., Prieto, M. S. C., \& Aznar, C. T. (2019). Virtual learning environments: Extending the technology acceptance model. Revista Electronica de Investigacion Educativa, 21(1), 1-12. https://doi.org/10.24320/REDIE.2019.21.E22.1866 